Нагапетян Артур Рубикович

аспирант, специалист отдела организации научно-исследовательской деятельности Школы экономики и менеджмента

Дальневосточного федерального университета

\section{КЛАСТЕРИЗАЦИЯ ВОЛАТИЛЬНОСТИ ДОХОДНОСТИ АКЦИЙ И ДИНАМИКА ДИВЕРСИФИКАЦИОННОГО ПОТЕНЦИАЛА НА РОССИЙСКОМ РЫНКЕ}

Аннотация:

В статье сформулировано понятие диверсификационного потенциала заданного множества активов, имеющее определяющее значение в механизме трансформации кластеризации волатильности доходности отдельных активов в кластеризацию на макроуровне и отражающее степень разнообразия их инвестиционных характеристик в восприятии инвесторов. Разработаны и применены методика расчета динамического индекса диверсификационного потенциала рынка на основе динамики попарных коэффициентов корреляции и явления кластеризации волатильности доходности активов, а также модель прогнозирования динамических коэффициентов реализованной корреляции между активами.

Ключевые слова

кластеризация волатильности на микроуровне, кластеризация волатильности на макроуровне, диверсификационный потенциал, гипотеза эффективного рынка (ГЭР), прогнозирование волатильности, арбитраж, разнообразие.
Nagapetyan Arthur Rubikovich

PhD student, Expert Research Activities Management Department, School of Economics and Management, Far Eastern Federal University

\section{STOCK RETURNS VOLATILITY CLUSTERING AND DIVERSIFICATION POTENTIAL DYNAMICS ON THE RUSSIAN MARKET}

Summary:

The article presents the concept of Diversification Potential for the portfolio. This concept is the main element in micro-level volatility clustering - macro-leve volatility clustering transformation mechanism. The au thor developed and applied methods to calculate the market's dynamic Diversification Potential Index (DPI) based on the dynamics of pairwise assets correlation coefficients and the phenomenon of assets volatility clustering, as well as the model to forecast the dynamic coefficients of assets realized correlation.

Keywords:

micro-level volatility clustering, macro-level volatility clustering, diversification potential, efficient market hypothesis (EMH), volatility forecasting, arbitrage, diversity.

С момента зарождения основных постулатов неоклассической фринансовой школы, при ее длительной эволюции и последующем обретении статуса современного экономического мейнстрима не перестает быть актуальным сформированный С. Россом вопрос о теоретическом описании механизма имплементации рыночной информации в цене актива [1]. Речь идет о взаимодействии большого количества отдельных экономических агентов, а также о том, как это приводит к ценообразованию активов, согласующемуся с положениями об информационной эффективности рынка и стимулах для поиска и обмена информационными потоками между ними [2].

На сегодняшний день можно констатировать следующее:

- Наблюдаются недостаточная разработанность теоретических конструкций, интерпретирующих природу явления кластеризации волатильности доходности акций, а также необходимость использования динамических подходов к интерпретации и прогнозированию волатильности и иных характеристик финансовых инструментов [3].

- Следует разрабатывать подходы к интерпретации динамики волатильности макроиндикаторов на финансовом рынке, их прогнозированию и осуществлять соответствующую политику со стороны системообразующих субъектов финансового рынка, в том числе фринансовых регуляторов [4].

- Важно выявлять элементы информационной неэффективности рынка и одновременно обосновывать причины и меры по ее снижению ввиду высокого уровня транзакционных издержек на российском финансовом рынке [5].

Приведенные суждения определяют актуальность исследования кластеризации волатильности доходности акций на российском рынке. Введем некоторые понятия и определения.

Кластеризация волатильности доходности активов (на микроуровне) - финансовая категория, определяемая взаимосвязанностью и взаимозависимостью значений волатильности в разные периоды, вызванная относительной неспособностью рынка обеспечить полную согласованную имплементацию накопившейся информации в цене актива. Предлагаемая фрормулировка отличается от существующих, в большей степени отражающих формализованные подходы к ее 
идентификации, представлением ее как финансовой категории, возникающей вследствие указанной неспособности рынка обеспечить имплементацию информации в цене.

Диверсификационный потенциал заданного множества активов - финансовая категория, представляющая максимально возможную степень снижения рисков вновь формируемых инвестиционных портфелей на основе динамики их попарных коэффрициентов корреляции и отражающая меру разнообразия их инвестиционных характеристик в восприятии инвесторов. В отличие от существующих подходов на основе рыночных индексов введенное понятие позволяет не только оценивать динамику волатильности на макроуровне, но и определять относительную важность общих макроэкономических условий функционирования на рынке в целом или в определенном секторе по сравнению с частными инвестиционными параметрами отдельно рассматриваемых активов.

Кластеризация волатильности доходности активов на макроуровне - финансовая категория, показывающая повышенные взаимосвязанность и взаимозависимость значений доходности рыночного портфеля во времени, вызванная снижением величины диверсификационного потенциала из-за внимания инвесторов в большей степени к общим макроэкономическим условиям функционирования, а не к параметрам отдельно взятых бумаг.

Применение предлагаемой методики расчета динамического индекса диверсификационного потенциала рынка (англ. - diversification potential index (DPI)) на основе динамики попарных коэффициентов корреляции и кластеризации волатильности доходности отдельно рассматриваемых активов позволяет в реальном времени оценивать максимально возможную степень снижения рисков вновь формируемых инвестиционных портфелей [6]. Индекс диверсификационного потенциала экономики на базе тех или иных активов предлагаем рассчитывать по формуле (1):

$$
\mathrm{DI}_{\mathrm{t}}=\sum_{\mathrm{i}>\mathrm{j}} \alpha_{1} \alpha_{2} \operatorname{cor}\left(\mathrm{a}_{\mathrm{i}}, \mathrm{a}_{\mathrm{j}}\right)_{\mathrm{t}} \text {. }
$$

Здесь $\mathrm{DI}_{\mathrm{t}}$ - индекс диверсификационного потенциала экономики в момент $\mathrm{t} ; \operatorname{cor}\left(\mathrm{a}_{\mathrm{i}}, \mathrm{a}_{\mathrm{j}}\right)_{\mathrm{t}}-$ коэффицциент корреляции между активами $\mathrm{a}_{1}$ и $\mathrm{a}_{2}$ в момент $\mathrm{t} ; \alpha_{1}$ и $\alpha_{2}-$ вес активов.

Анализ DPI на наличие феномена кластеризации волатильности дает положительный результат. Это в том числе служит косвенным подтверждением истинности предложенного механизма трансформации явления кластеризации волатильности доходности с отдельных активов в кластеризацию волатильности доходности активов на макроуровне.

DPI может быть интерпретирован как среднее значение динамических условных попарных коэффициентов корреляции. Следовательно, чем ниже корреляция, тем выше диверсификационный потенциал. На рисунке 1 представлена динамика значений индекса диверсификационного потенциала рынка и индекса Московской биржи в период с 2012 по 2017 г.

$$
\text { MICEX_and_DPI }
$$

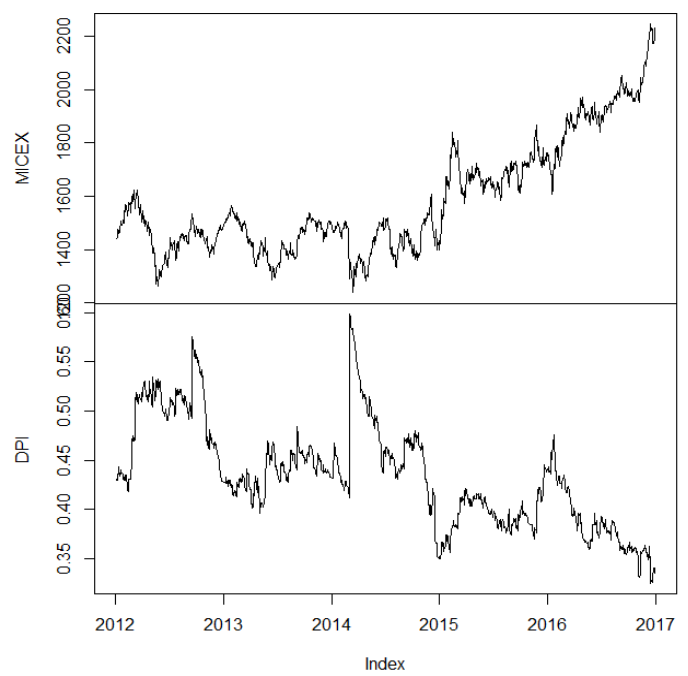

Рисунок 1 - Динамика значений DPI и индекса Московской биржи с 2012 по 2017 г.

Корреляция между данными показателями, как и предсказывается в гипотезе, отрицательная: -0,73. Однако важно также указать, что данные коинтегрированы, что проверено с помощью интегрированного расширенного теста Дики - Фуллера (англ. - cointegrated augmented Dickey Fuller test (CADF)). Значения статистики CADF: -3.57 и -4.21 соответственно; р-значения: 0,03 и 0,01. Следует отметить, что сегодняшние величины DPI существенно влияют на будущие цифры индекса Московской биржи даже с учетом рассмотрения лага последнего.

На основе гетерогенной авторегрессионной модели реализованной волатильности (англ. heterogenous autoregressive model of realized volatility (HAR-RV)) мы разработали модель прогнозирования реализованной корреляции. Она позволяет прогнозировать будущие значения как 
предлагаемого динамического индекса диверсификационного потенциала, так и любых других показателей оценки финансового риска, при расчете которых используется коэфффициент корреляции. Кроме того, в отличие от модели динамической условной корреляции она основывается на непосредственно реализованных значениях корреляции за прошлые периоды и имеет более ясную экономическую интерпретацию.

Модель гетерогенной авторегрессионной реализованной волатильности получила развитие в работах P. Wang, H. Liu, A. Щербы [7]. В рамках разработки и применения модели прогнозирования динамических коэффициентов реализованной корреляции между активами, в том числе для предсказывания величин волатильности портфелей активов, построения динамических бета-коэффициентов и иных подходов к оценке финансовых рисков, предлагаем использовать описанную авторскую модель гетерогенной авторегрессионной реализованной волатильности. Данный подход представлен в формуле (2):

$$
\operatorname{cor}(\mathrm{i}, \mathrm{j})_{\mathrm{t}+1 \mathrm{~d}}^{(\mathrm{d})}=\mathrm{c}+\beta^{(\mathrm{d})} \operatorname{Rcor}(\mathrm{i}, \mathrm{j})_{\mathrm{t}}^{(\mathrm{d})}+\beta^{(\mathrm{w})} \operatorname{Rcor}(\mathrm{i}, \mathrm{j})_{\mathrm{t}}^{(\mathrm{w})}+\beta^{(\mathrm{m})} \operatorname{Rcor}(\mathrm{i}, \mathrm{j})_{\mathrm{t}}^{(\mathrm{m})}+\varepsilon_{\mathrm{t}},
$$

где $\operatorname{cor}(\mathrm{i}, \mathrm{j})_{\mathrm{t}+1 \mathrm{~d}}^{(\mathrm{d})}$ - оценка предсказания корреляции активов і и j на один день; $\operatorname{Rcor}_{\mathrm{t}}^{(\mathrm{d})}$ - оценка величины реализованной корреляции; $\mathrm{w}=5 ; \mathrm{m}=22 ; \operatorname{Rcor}_{\mathrm{t}}^{(\mathrm{n})}=\left(\operatorname{Rcor}_{\mathrm{t}}+\cdots+\mathrm{Rcor}_{\mathrm{t}-\mathrm{n}+1}\right) / \mathrm{n} ; \varepsilon_{\mathrm{t}}-$ ошибка модели, подчиняющаяся закону «белого шума».

Можно установить величину DPI по предложенной методике на основе реализованной попарной корреляции вместо использования условной попарной корреляции на базе модели динамической условной корреляции. На рисунке 2 отражена динамика индекса диверсификационного потенциала рынка, определенного с помощью попарных коэффициентов реализованной корреляции в период с 2014 по 2017 г. Если применять подход с введением скользящего среднего в форме 22 предыдущих рабочих дней, то графики динамики DPI, рассчитанные на основе методов условной корреляции и реализованной корреляции, будут более похожи друг на друга (рисунок 3).

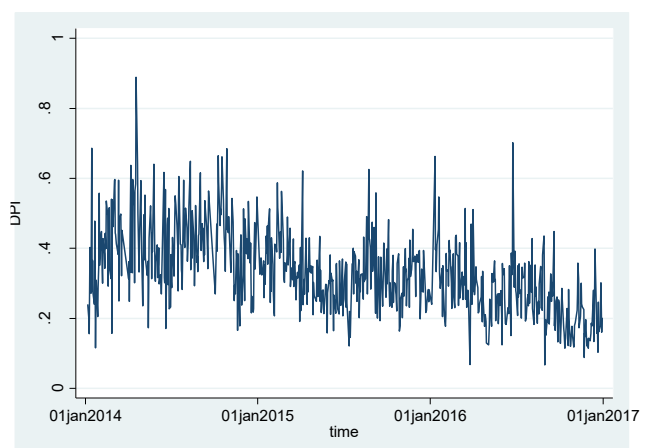

Рисунок 2 - Динамика DPI рынка с использованием попарных коэффрициентов реализованной корреляции в период с 2014 по 2017 г.

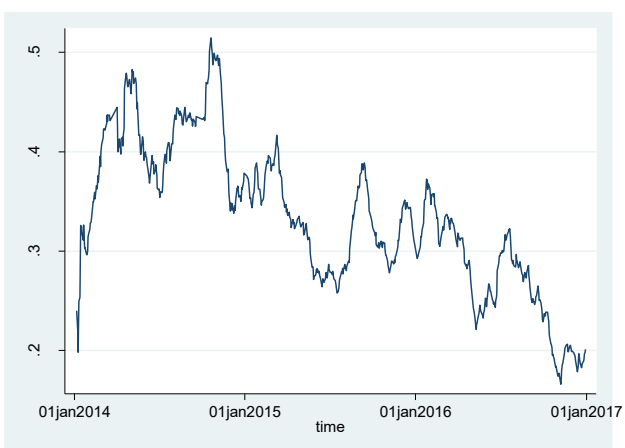

Рисунок 3 - Скользящее среднее $(22,1,0)$ динамики DPI рынка с использованием попарных коэффициентов реализованной корреляции в период с 2014 по 2017 г.

На основе модели гетерогенной авторегрессионной реализованной корреляции можно прогнозировать будущие значения корреляции доходности активов. В таблице 1 приведены величины коэфффициентов регрессии при применении модели гомогенной авторегрессионной реализованной корреляции для прогнозирования значений индекса диверсификационного потенциала с использованием попарных коэффициентов реализованной корреляции.

Таблица 1 - Значения коэффрициентов регрессии $\boldsymbol{\beta}^{(\mathrm{d})}, \boldsymbol{\beta}^{(\mathrm{w})}, \boldsymbol{\beta}^{(\mathrm{m})}$ в модели гетерогенной авторегрессионной реализованной корреляции для прогнозирования DPI

\begin{tabular}{|c|c|c|c|}
\hline Показатель & $\operatorname{DPI}\left(\operatorname{DPI}^{(\mathbf{d})}\right)$ & $\operatorname{DPI}\left(D P I^{(d)}, D^{\left(I^{(w)}\right.}\right)$ & $\operatorname{DPI}\left(\mathrm{DPI}^{(\mathrm{d})}, \mathrm{DPI}^{(\mathrm{w})}, \mathrm{DPI}^{(\mathrm{m})}\right)$ \\
\hline $\mathrm{DPI}^{(\mathrm{d})}$ & $\begin{array}{c}0.473 \\
(13.14)^{\star *}\end{array}$ & $\begin{array}{l}-0.243 \\
(5.95)^{\star *}\end{array}$ & $\begin{array}{l}-0.243 \\
(5.94)^{* *}\end{array}$ \\
\hline $\mathrm{DPI}^{(\mathrm{w})}$ & 1.01 .7 & $\begin{array}{c}1.197 \\
(22.79)^{* *}\end{array}$ & $\begin{array}{c}1.183 \\
(15.94)^{\star *}\end{array}$ \\
\hline $\mathrm{DPI}^{(\mathrm{m})}$ & - & - & $\begin{array}{c}0.020 \\
(0.27)^{\star}\end{array}$ \\
\hline cons & $\begin{array}{c}0.170 \\
(13.51)^{* *}\end{array}$ & $\begin{array}{c}0.009 \\
(0.80)^{*}\end{array}$ & $\begin{array}{c}0.007 \\
(0.54)^{*}\end{array}$ \\
\hline $\mathrm{R}^{2}$ & 0.23 & 0.60 & 0.60 \\
\hline $\mathrm{N}$ & 570 & 570 & 570 \\
\hline
\end{tabular}


В отличие от подхода, основанного на использовании модели динамической условной корреляции, в модели гетерогенной авторегрессионной реализованной корреляции используются наблюдаемые значения корреляции внутридневных данных. Результаты согласуются с отраженными на рисунках цифрами, согласно которым наиболее точный прогноз обеспечивает средняя величина реализованной корреляции за предыдущие 5 дней. Также видна отрицательная связь значений реализованной корреляции с аналогичными данными за последующий день. В рассмотренном случае коэффициент регрессии при переменной, характеризующей среднюю корреляцию за предыдущие 22 рабочих дня, оказался незначимым на 5 \%-м уровне значимости. Однако его сохранение в модели необходимо ввиду большого теоретического значения и для точности оценки параметров при остальных переменных.

Рассмотрим итоги. Предложено определение, уточняющее понятие кластеризации волатильности доходности на микроуровне как финансовой категории, отражающее природу предпосылок ее возникновения, демонстрирующее направления совершенствования подходов к оценке волатильности финансовых активов и формированию арбитражных стратегий в контексте повышения информационной эфффективности рынка. Приведена формулировка диверсификационного потенциала заданного множества активов, имеющего определяющее значение в механизме трансформации кластеризации волатильности доходности отдельных активов в кластеризацию на макроуровне и подразумевающего разнообразие их инвестиционных характеристик в восприятии инвесторов [8]. Разработаны и применены методика расчета динамического индекса диверсификационного потенциала рынка на основе динамики попарных коэффициентов корреляции и явления кластеризации волатильности доходности активов, а также модель прогнозирования динамических коэффициентов реализованной корреляции между активами.

Полученные результаты могут быть применены в большинстве подходов к анализу рисков финансовых инструментов, для реализации которых необходимы точные данные ожидаемых значений волатильности в будущих периодах (например, VaR - value at risk), а также для совершенствования методов оценки и информационной эффеективности рынков и поиска арбитражных стратегий, направленных на ее повышение на основе требуемого равенства односторонних коэффициентов чувствительности портфелей финансовых активов к положительным и отрицательным рыночным изменениям. Введенное понятие диверсификационного потенциала заданного множества активов (рынка) и методика ее расчета могут быть полезны при исследовании инвестиционной привлекательности рынков, прогнозировании спроса на финансовые ресурсы (для фринансового регулятора), а также предсказании динамики рыночного индекса.

\section{Ссылки:}

1. Ross S.A. The arbitrage theory of capital asset pricing // Journal of Economic Theory. 1976. Vol. 13, no. 3. P. 341-360.

2. Нагапетян А.Р., Рубинштейн Е.Д., Урумова Ф.М. Развитие современной портфельной теории: деформации ценообразования и арбитраж // Вестник ИЭ РАН. 2015. № 3.

3. Субботин А.В. Моделирование волатильности: от условной гетероскедастичности к каскадам на множественных горизонтах // Прикладная эконометрика. 2009. № 3. С. 94-138.

4. Асатуров К., Теплова Т. Построение коэффициентов хеджирования для высоколиквидных акций российского рынка на основе моделей класса GARCH // Экономика и математические методы. 2014. Т. 50, № 1. С. 37-54.

5. Лебедев О.В. Концепция рыночной эффеективности: теоретическое и эмпирическое обоснование и роль в создании стоимости // Корпоративные финансы. 2011. № 2. С. 82-91.

6. Bollerslev T. Generalized autoregressive conditional heteroskedasticity // Journal of Econometrics. 1986. Vol. 31, no. 3. P. 307-327 ; Engle R. Autoregressive conditional heteroscedasticity with estimates of variance of United Kingdom inflation // Econometrica. 1982. Vol. 50. P. 987-1008; Idem. Dynamic conditional correlation - a simple class of multivariate GARCH models // Journal of Business and Economic Statistics. 2002. Vol. 20, no. 3. P. 339-350.

7. Wang P. Modeling and forecasting of realized volatility based on high-frequency data: Evidence from FTSE-100 index. Hanken, 2009 ; Щерба A. Сравнение моделей оценок VAR на интервалах прогнозирования разной срочности для акций российского фондового рынка // Прикладная эконометрика. 2011. № 4 (24). С. 58-70.

8. Mandelbrot B. The variation of certain speculative prices // Journal of Business. 1963. Vol. 36. P. 394-419.

\section{References}

Asaturov, K \& Teplova, T 2014, 'Construction of hedging coefficients for highly liquid shares of the Russian market on the basis of GARCH models', Ekonomika i matematicheskiye metody, vol. 50, no. 1, pp. 37-54, (in Russian).

Bollerslev, T 1986, 'Generalized autoregressive conditional heteroskedasticity', Journal of Econometrics, vol. 31, no. 3, pp. 307-327, https://doi.org/10.1016/0304-4076(86)90063-1.

Engle, R 1982, 'Autoregressive conditional heteroscedasticity with estimates of variance of United Kingdom inflation', Econometrica, vol. 50, pp. 987-1008, https://doi.org/10.2307/1912773.

Engle, R 2002, 'Dynamic conditional correlation - a simple class of multivariate GARCH models', Journal of Business and Economic Statistics, vol. 20, no. 3, pp. 339-350, https://doi.org/10.1198/073500102288618487.

Lebedev, OV 2011, 'The concept of market efficiency: theoretical and empirical justification and role in creating value', Korporativnyye finansy, no. 2, pp. 82-91, (in Russian). 
Mandelbrot, B 1963, 'The variation of certain speculative prices', Journal of Business, vol. 36, pp. 394-419, https://doi.org/10.1086/294632.

Nagapetyan, AR, Rubinshtein, ED \& Urumova, FM 2015, 'The development of modern portfolio theory: pricing deformation and arbitration', Vestnik IE RAN, no. 3, (in Russian).

Ross, SA 1976, 'The arbitrage theory of capital asset pricing', Journal of Economic Theory, vol. 13, no. 3, pp. 341-360, https://doi.org/10.1016/0022-0531(76)90046-6.

Shcherba, A 2011, 'Comparison of VAR estimation models while forecasting the different maturities for shares of the Russian stock market', Prikladnaya ekonometrika, no. 4 (24), pp. 58-70, (in Russian).

Subbotin, AV 2009, 'Volatility models: from conditional heteroscedasticity to cascades at multiple horizons', Prikladnaya ekonometrika, no. 3, pp. 94-138, (in Russian).

Wang, P 2009, Modeling and forecasting of realized volatility based on high-frequency data: Evidence from FTSE-100 index, Hanken. 